\title{
Potential Energy Surface of NO on Pt(997): Adsorbed States and Surface Diffusion
}

\author{
N. Tsukahara ${ }^{1}$ and J. Yoshinobu ${ }^{2}$ \\ ${ }^{1}$ Department of Advanced Material Sciences, The University of Tokyo, Kashiwa, Chiba 277-8561, Japan \\ ${ }^{2}$ The Institute for Solid State Physics, The University of Tokyo, Kashiwa, Chiba 277-8581, Japan
}

Correspondence should be addressed to J. Yoshinobu, yoshinobu@issp.u-tokyo.ac.jp

Received 31 May 2011; Revised 3 August 2011; Accepted 7 August 2011

Academic Editor: António Varandas

Copyright ( $\odot 2012$ N. Tsukahara and J. Yoshinobu. This is an open access article distributed under the Creative Commons Attribution License, which permits unrestricted use, distribution, and reproduction in any medium, provided the original work is properly cited.

The potential energy surface (PES) of NO on $\mathrm{Pt}(997)$ has been elucidated: the adsorption states and diffusion processes of NO on $\mathrm{Pt}(997)$ at low coverage were investigated by using infrared reflection absorption spectroscopy (IRAS) and scanning tunneling microscopy (STM). When NO molecules adsorb on a surface at a low temperature $(11 \mathrm{~K})$, each molecule transiently migrates on the surface from the first impact point to a possible adsorption site. We found that there are four stable adsorption sites for NO on Pt(997): a bridge site of the upper step, an fcc- (or hcp-) hollow site of the terrace, an on-top site of the terrace, and an fcc-hollow site of the lower step. At higher temperatures above $45 \mathrm{~K}$, NO molecules start to migrate thermally to more stable adsorption sites on a terrace, and they are finally trapped at the bridge sites of the step, which are the most stable among the four sites.

\section{Introduction}

The elucidation of potential energy surface (PES) of adsorbed molecules on metal surfaces is quite important. In particular, surface diffusion of adsorbed atoms and molecules plays a vital role in various surface dynamical processes, such as heterogeneous chemical reactions, formation of self-assembled structures, and single atom manipulation by a scanning probe microscope [1]. Since an adsorbed molecule must encounter a reaction partner before a chemical reaction takes place, surface diffusion could be a rate-limiting process for surface chemical reactions. In particular, the adsorption of carbon monoxide (CO) and nitric monoxide (NO) on transition metal surfaces is a basic model for investigating the catalytic reaction on the automobile threeway catalyst, where the PES for CO and NO governs the migration of the molecules. Therefore, not only surface diffusion but also the adsorption states of $\mathrm{CO}$ and $\mathrm{NO}$ are important. So far, many studies of $\mathrm{CO}$ and $\mathrm{NO}$ on transition metal surfaces have been reported; $\mathrm{NO}$ adsorption on $\operatorname{Pt}(111)$ is one of the fundamental systems and there are many studies [2-10]. In addition, surface defects are often stable adsorption sites and active sites for NO dissociation
[11-13]. Therefore, the adsorption states and diffusion process of NO on a stepped Pt surface are even more important than that on a flat Pt surface.

In this review article, the adsorption states and the diffusion process of $\mathrm{NO}$ molecules on $\mathrm{Pt}(997)$ are discussed based on our recent investigations by infrared reflection absorption spectroscopy (IRAS) and scanning tunneling microscopy (STM).

Surface diffusion is classified into "thermal" and "transient" diffusion [1]. Thermal diffusion consists of stochastic site-to-site hopping motions of adsorbates driven by thermal fluctuation (Brownian motion). Based on Arrhenius-type rate theory, the hopping rate, $k$, is defined as follows:

$$
k=A \exp \left(-\frac{E}{k_{B} T}\right)
$$

where $E, T$, and $A$ are the activation barriers to site-to-site hopping, substrate temperature, and preexponential factor, respectively. On the other hand, transient diffusion is the inevitable process upon adsorption $[1,14]$. When an atom or a molecule is adsorbed on a surface, adsorption energy must be dissipated. Excitations of various vibrations of a 


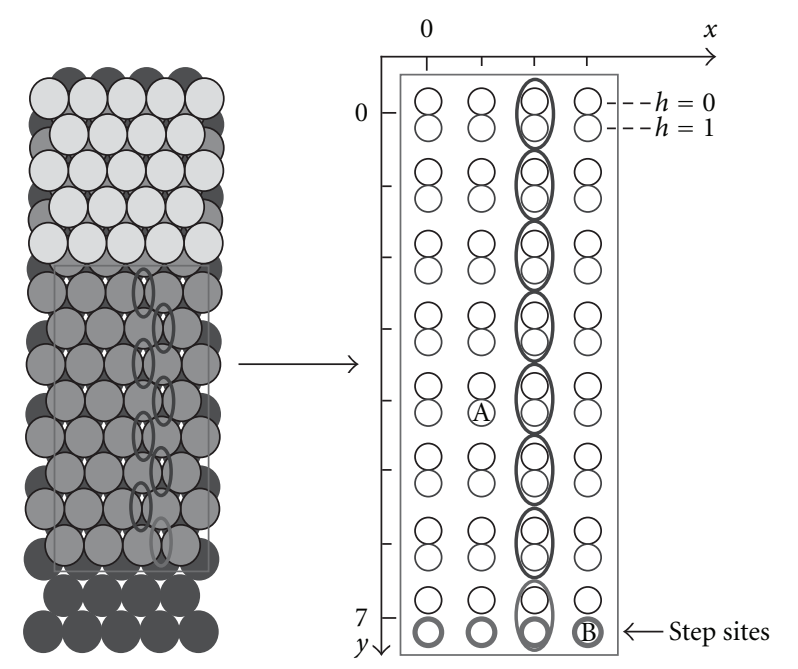

(a) Real Pt(997) lattice

(b) A lattice used in the KMC simulations

FIgURe 1: (a) A schematic drawing of Pt(997). (b) A lattice used in the present KMC simulation. The lattice size is about $280 \times 280 \AA^{2}$. Circles of thin lines represent the fcc-and hcp-hollow sites on the terrace, and circles of heavy lines represent the bridge sites on the step, respectively. Although the lattice of the (111) terrace is hexagonal, we virtually deformed the lattice into a tetragonal one to assign adsorption sites with Cartesian coordinates. The coordinates of adsorption sites on the terrace and the step are represented by $(x, y, h, s)$ and $(x, s)$, respectively. For example, the coordinates labeled "A" and "B" in the lattice are represented as $(1,4,1,0)$ and $(3,0)$, respectively [15].

substrate and an adsorbed molecule, electron-hole pair excitations, and transient motions of an adsorbate are possible dissipation paths. The transient diffusion is the translational migration of an adsorbate driven by its adsorption energy. This depends not on the substrate temperature, but on the interaction between adsorbates and the substrate. Energy dissipation of chemisorbed species is more efficient than that of physisorbed species because of the mixing of the electronic states in chemisorption. In addition, the PES of chemisorbed species is more complicated than that of physisorbed species. Therefore, chemisorbed species have a shorter diffusion length in transient diffusion than physisorbed species [1].

\section{Experimental and Analysis Methods}

All IRAS and STM experiments were carried out in an ultrahigh vacuum chamber. The base pressure of the chamber is less than $1 \times 10^{-10}$ Torr.

A clean $\operatorname{Pt}(997)$ surface was carefully prepared by repeated cycles of $\mathrm{Ne}$ ion sputtering and annealing procedures in a preparation chamber. The $\mathrm{Pt}(997)$ surface has a periodic step-terrace structure (Figure 1(a)). The terrace has a (111) close-packed structure composed of nine rows of platinum atoms, and the monoatomic step is formed by a (111) microfacet. In the final cleaning procedure, the sample was exposed to $2 \times 10^{-7}$ Torr $\mathrm{O}_{2}$ with annealing at $1000 \mathrm{~K}$ to remove trace carbon contamination. Subsequently, the sample was flashed to $1300 \mathrm{~K}$. The surface contaminations, mainly carbon, were reduced by repeated cycles of this oxidation procedure. The cleanness of the $\operatorname{Pt}(997)$ surface was confirmed by using low energy electron diffraction (LEED) and STM.
The sample was transferred to a measurement chamber in which the sample holder was connected to a liquid $\mathrm{He} /$ liquid $\mathrm{N}_{2}$ reservoir and surrounded by triple thermal shields. Gaseous NO molecules at $300 \mathrm{~K}\left(k_{B} T \sim 26 \mathrm{meV}\right)$ were introduced onto the cold sample surface through a pulse gas dosing system, at an angle of about $45^{\circ}$ from the surface normal direction.

In IRAS measurements, the resolution and the number of accumulations were $4 \mathrm{~cm}^{-1}$ and 500 scans, respectively. In time-resolved IRAS (TR-IRAS) measurements, the resolution was $4 \mathrm{~cm}^{-1}$, the same as with the normal IRAS measurements. The measurement time of each spectrum was controlled by the number of accumulations. In this study, it was from 250 to 500 scans, which corresponds to a time increment from 50 to $100 \mathrm{~s}$. When the valve of the pulse gas dosing system was opened, the time-resolved measurements were started, and a series of IRAS spectra were acquired as a function of the elapsed time. The exposure of NO molecules was controlled by the duration time of the pulse gas dosing system. The duration time (the open time of the valve) was in the order of ms (typically $2 \sim 5 \mathrm{~ms}$ ) and much shorter than the measurement time. Details of the experiments are described in our previous papers [15-17].

In order to analyze the results of TR-IRAS in the range from 100 to $110 \mathrm{~K}$, where the long range diffusion of NO across the terrace and the island formation occurs, Kinetic Monte Carlo (KMC) simulation was carried out. Here, the procedure of the present KMC simulation is described. The KMC simulation is performed with the lattice shown in Figure 1 with a periodic boundary condition. The migration of the adsorbed NO molecules is simulated using a lattice gas model $[18,19]$. On the terraces and the steps, the arrays $c(x, y, h, s)$ and $c_{s}(x, s)$ are defined, and the value of each 


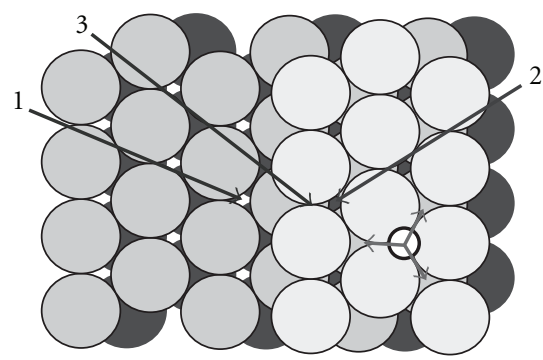

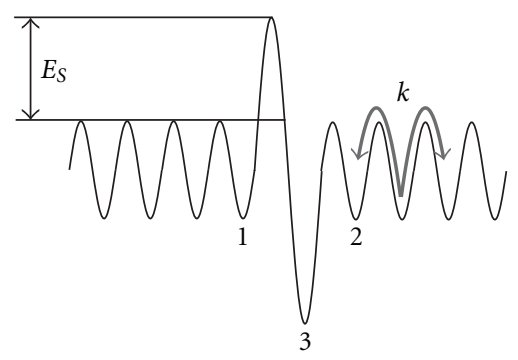

(a)

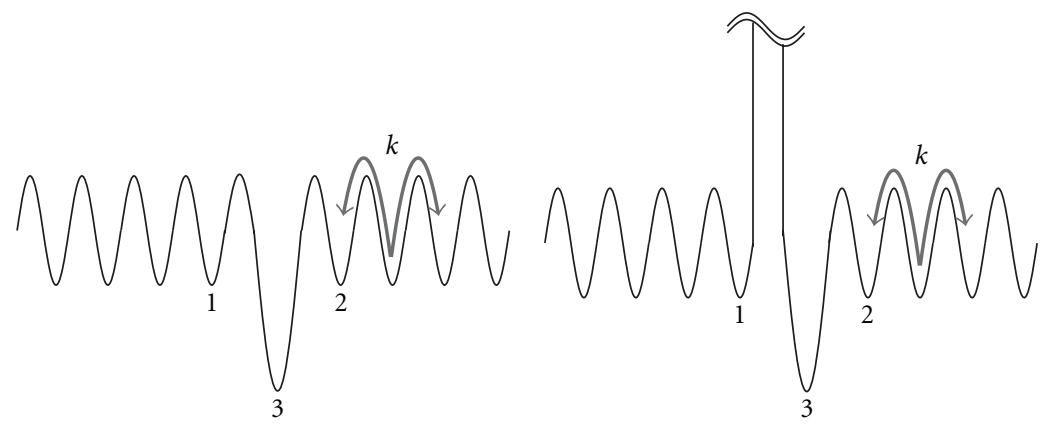

(b) Model A; $E_{S}=0$

(c) Model B; $E_{S}=\infty$

FIgURE 2: (a) A schematic drawing of Pt(997) around the step and its PES with ES barrier $E_{S}$. Sites 1, 2, and 3 are the HS site, HT site next to the BS site, and BS site, respectively. $k$ is the NO does not adsorb, is the hcp-hollow site next to the HS site. $k$ is the rate constant to migrate from a hollow site to a neighboring hollow site on the terrace. (b), (c) Models of PES in the KMC simulation. In model A, the rate constant from site 1 to site 3 is assumed to be the same as that from site 2 to site 3 . In model B, the rate constant from site 1 to site 3 is assumed to be zero (infinite barrier).

element of the arrays is either 0 (empty) or 1 (occupied). In order to characterize the hopping processes of adsorbed $\mathrm{NO}$, a rate constant $k$ is assigned to each hopping motion. An NO molecule at a hollow site on the (111) terrace (HT species) migrates to a nearest neighbor HT site via a bridge site [13]. Therefore, the HT species has three nearest neighbors, meaning that three rate constants are required for one HT species. In this model, all the rate constants of hopping from the hollow to the nearest neighbor are assumed to be the same. A similar assumption was adopted in the previous study of $\mathrm{CO}$ on stepped $\mathrm{Pt}(111)$ surfaces [20-22].

In addition, the Ehrlich-Schwoebel (ES) effect [23, 24], which is shown in Figure 2, should be considered in the $\mathrm{KMC}$ simulation. If an atom or molecule approaches from a lower or an upper terrace to a step region, the PES across the step shows an asymmetric profile. In the case of $\mathrm{CO}$ on a stepped $\mathrm{Pt}(111)$ surface, a very large barrier on the route from a lower terrace to an upper step must be considered [20-22]. In order to incorporate the ES effect (Figure 2(a)) simply, two cases are considered: model A (Figure 2(b)) has no Schwoebel barrier, while model B (Figure 2(c)) has an infinite barrier. In model A, HT species on a lower terrace climb up to an upper step site. On the other hand, in model $\mathrm{B}, \mathrm{HT}$ species on a lower terrace do not climb up to the upper step and are reflected by the ES barrier. Thus, the molecules can reach the step sites from one direction exclusively.

An adsorbate-adsorbate interaction was also taken into account in the simulation because HT species on $\mathrm{Pt}(111)$ form a $p(2 \times 2)$ structure [2-10]. Therefore, when they form the $p(2 \times 2)$ structure, energy modifications, which are expressed by multiplying the rate constant $k$ by a Boltzmann factor $\exp \left(-E_{\text {int }} / k_{B} T\right)$, must be considered. $E_{\text {int }}$ is the interaction energy. The interaction is assumed only to be the factor lowering the potential energy minimum of the HT species [25-29]. In addition, all the rate constants whose processes reduce the distance between HT species to less than $5.54 \AA$ are zero. This procedure represents the repulsive interaction between $\mathrm{NO}$ molecules. The value of $5.54 \AA$ corresponds to the distance between hollow species in the $p(2 \times 2)$ structure on $\operatorname{Pt}(111)$.

The initial $(t=0)$ distribution of NO molecules was determined stochastically. The details are described in our previous report [15]. Once the initial distribution was determined, repeated cycles of the standard KMC procedure were carried out until the elapsed time exceeded a preset value. In this study, 2000-100,000 cycles of the KMC step were carried out. Parameters $r_{s}, k$, and $E_{\text {int }}$ were determined to fit the simulation with the experiment.

\section{Adsorption States and Diffusion Kinetics}

3.1. Adsorption States of NO Molecules on Pt(997). Figure 3 shows a series of IRAS spectra of NO molecules (0.017 ML) on $\operatorname{Pt}(997)$ as a function of substrate temperature. In Figure 3(a), four peaks are observed at $11 \mathrm{~K}$. All peaks are derived from the stretching mode $v(\mathrm{~N}-\mathrm{O})$; there are four stable adsorption sites on $\mathrm{Pt}(997)$. Table 1 shows the assignment of the NO adsorption sites. The peaks at 1483 and $1689 \mathrm{~cm}^{-1}$ are observed in the previous vibrational studies of $\mathrm{NO}$ on a flat $\mathrm{Pt}(111)$ at low temperatures and are assigned to the NO molecules at the threefold hollow 
TAble 1: Adsorbed states of NO on Pt(997). Observed and calculated energies are shown. * Details of DFT calculations are shown in our previous report [16].

\begin{tabular}{|c|c|c|c|c|}
\hline \multirow{2}{*}{ Adsorption site } & \multicolumn{2}{|c|}{$v(\mathrm{~N}-\mathrm{O})\left(\mathrm{cm}^{-1}\right)$} & \multirow{2}{*}{ Adsorption energy (eV) } & \multirow{2}{*}{ Abbreviation } \\
\hline & Observed & Calculated* & & \\
\hline \multirow{2}{*}{ Hollow (terrace) } & \multirow{2}{*}{$1482 \sim 1484$} & $1512(\mathrm{fcc})$ & 2.09 (fcc) & \multirow{2}{*}{$\mathrm{HT}$} \\
\hline & & 1539 (hcp) & $1.92(\mathrm{hcp})$ & \\
\hline On-top (terrace) & $1689 \sim 1690$ & 1683 & 1.61 & OT \\
\hline Bridge (upper step) & $1610 \sim 1630$ & 1607 & 2.49 & BS \\
\hline Hcp-hollow (lower step) & - & 1188 & 0.87 & - \\
\hline Fcc-hollow (lower step) & $1385 \sim 1396$ & - & - & HS \\
\hline
\end{tabular}

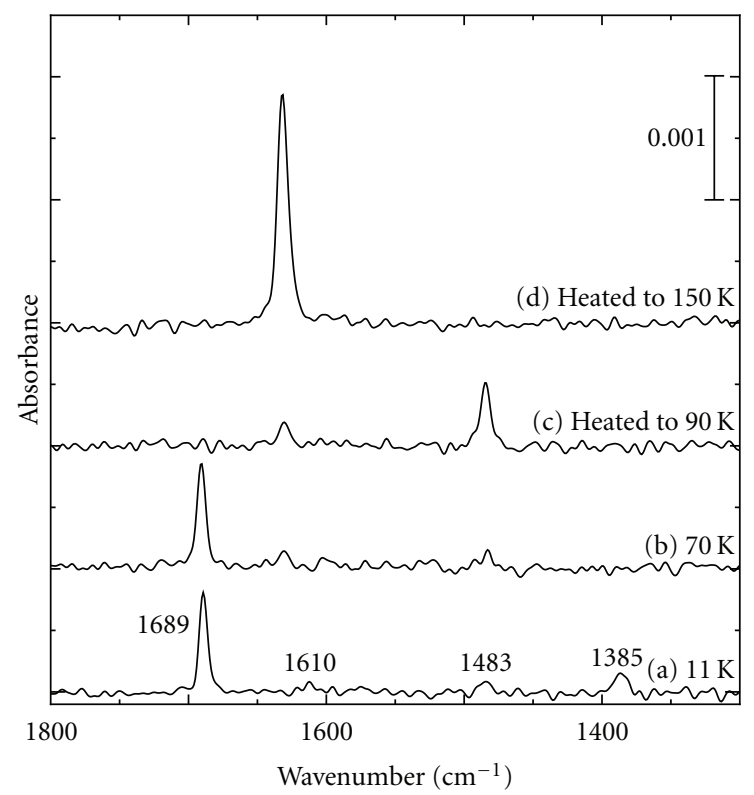

Figure 3: IRAS spectra of 0.017 ML NO on Pt(997) as a function of substrate temperature. (a) A spectrum after an $\mathrm{NO}$ gas injection at $11 \mathrm{~K}$. (b) A spectrum after an $\mathrm{NO}$ gas injection at $70 \mathrm{~K}$. (c) After heating the substrate from $70 \mathrm{~K}$ to $90 \mathrm{~K}$, and cooling down to $70 \mathrm{~K}$. (d) After heating the substrate from $70 \mathrm{~K}$ to $150 \mathrm{~K}$, and cooling down to $70 \mathrm{~K}$.

site and on-top site (HT and OT species) [6-10]. Other peaks at 1385 and $1610 \mathrm{~cm}^{-1}$ have not been observed at NO on a flat $\mathrm{Pt}(111)$ surface; thus, they are related to the monoatomic step structure of $\mathrm{Pt}(997)$. By increasing the substrate temperature (Figures $3(\mathrm{~b})-3(\mathrm{~d})$ ), the number of peaks is decreased. This is caused by the thermal diffusion of adsorbed NO. Adsorbed NO molecules thermally migrate on a surface to more stable adsorption sites. Thermal diffusion is discussed after the assignment of the four peaks in Figure 3(a).

Figure 4(a) shows an STM image of NO molecules (0.2 ML) on $\mathrm{Pt}(997)$ at $86 \mathrm{~K}$. Adsorbed $\mathrm{NO}$ molecules are observed as protrusions on $\mathrm{Pt}(997)$. Since the substrate temperature in the STM result is almost the same as that in the IRAS result of Figure 3(c), the STM image should contain two NO adsorption species $\left(1483\right.$ and $\left.1610 \mathrm{~cm}^{-1}\right)$. The protrusions on the terrace are HT species and they form a $p(2 \times 2)$ structure on the terrace, giving the $1483 \mathrm{~cm}^{-1}$ peak. Therefore, the protrusions on the step edge correspond to the peak at $1610 \mathrm{~cm}^{-1}$. By drawing a registry grid on the STM image, the protrusions on the step edge are located at the twofold bridge sites. Therefore, the stable adsorption site for NO molecules on the step edge is a twofold bridge site, and the peak at $1610 \mathrm{~cm}^{-1}$ is assigned to $\mathrm{NO}$ at the bridge site of the upper step (BS species). Compared to the previous DFT calculation, the BS species is the most stable species, and the stretching frequency is $1607 \mathrm{~cm}^{-1}$, which is in good agreement with the experimental results [16]. The other peak at $1385 \mathrm{~cm}^{-1}$, which appears at $11 \mathrm{~K}$, is assigned to the NO at the HS species shown in Figure 4(b). From the DFT calculation, there is a stable adsorption site at an HS (hcp) site as shown in Figure 4(b), and its calculated stretching frequency is $1188 \mathrm{~cm}^{-1}$ [16]. This is smaller than the experimental result. We think that not the HS (hcp) site but the HS site is stable for the NO, which has stretching frequency of $1385 \mathrm{~cm}^{-1}$. We think that HS species interact with the step edge more strongly than HT species. The hybridization between the Pt $5 \mathrm{~d}$ state of the step edge and the NO $2 \pi$ orbital increases the back donation of the electron from the Pt substrate to the $2 \pi$ orbital compared to the NO on the terrace, and the electron back donation lowers the stretching frequency.

At $70 \mathrm{~K}, \mathrm{OT}, \mathrm{HT}$, and BS species are observed by IRAS (Figure 3(b)), and HS species is not observed. This suggests that the HS species is the least stable among the four. By heating the substrate to $90 \mathrm{~K}$, thermal diffusion of $\mathrm{NO}$ from OT sites is now activated. The peak of OT species disappears and the intensities of others are increased (Figure 3(c)). By heating the substrate to $150 \mathrm{~K}$, all adsorbed NO molecules migrate to the BS sites (Figure 3(d)). Therefore, the most stable adsorption site for NO is the BS site, and the 2nd and 3rd most stable sites are the HT and OT sites, respectively. These results are in good agreement with the previous DFT calculation [16] and are summarized in Table 1. shows a series of TR-IRAS spectra of NO on $\mathrm{Pt}(997)$ at $76 \mathrm{~K}$, 


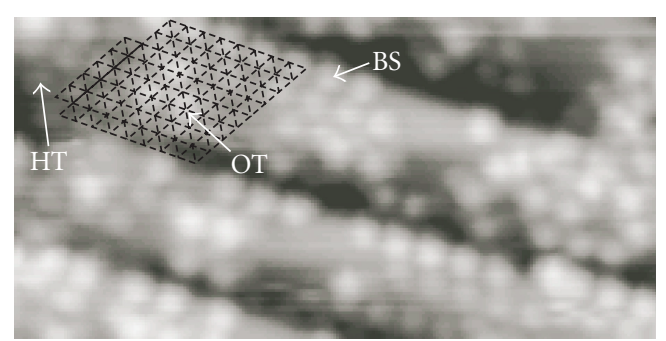

(a)

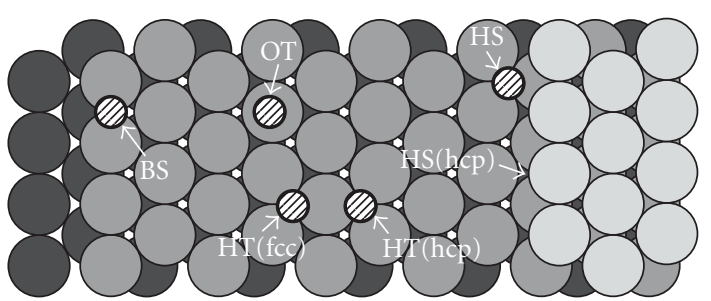

(b)

Figure 4: (a) An STM image of $0.2 \mathrm{ML}$ NO on Pt(997) at $86 \mathrm{~K} . V_{s}=-0.1 \mathrm{~V}, I_{t}=200 \mathrm{pA}, 11 \mathrm{~nm} \times 5.5 \mathrm{~nm}$. The registry mesh is drawn based on adsorbed NO molecules on the terrace as a reference. The intersections correspond to the position of Pt atoms. (b) A schematic drawing of the adsorption sites of the NO on Pt(997). HT (fcc and hcp), OT, BS, and HS species are energetically stable. However, an HS (hcp) site is not stable.

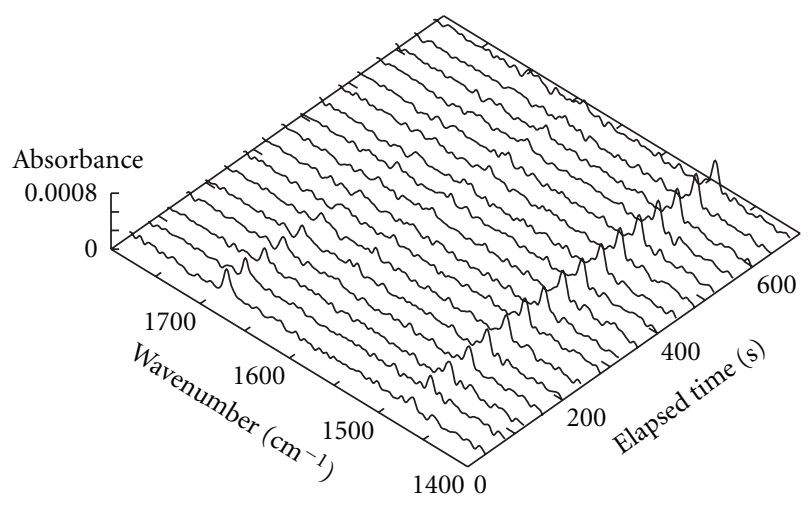

(a)

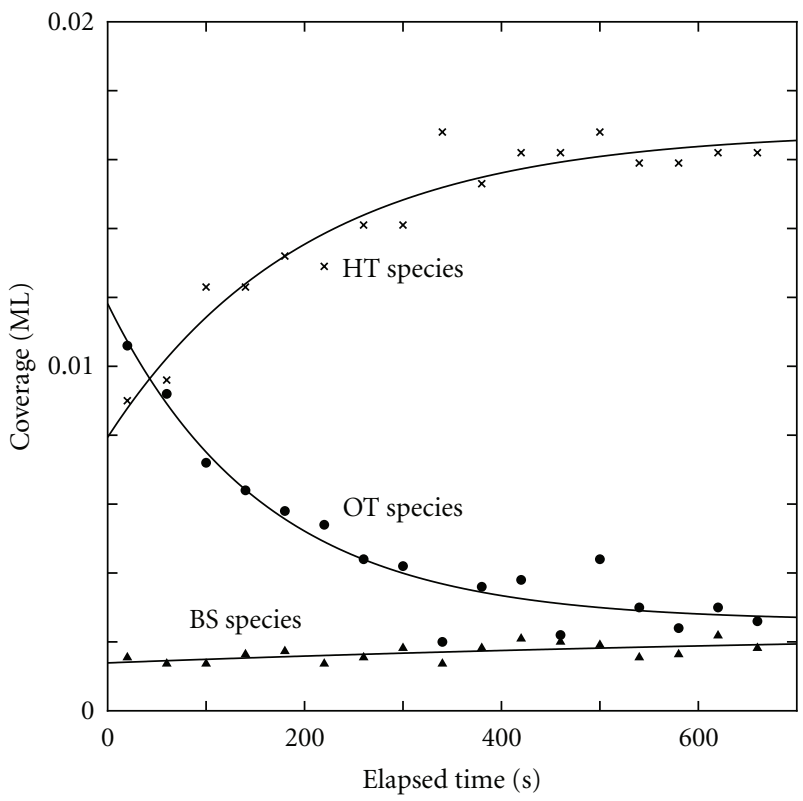

(b)

FIgURE 5: (a) A series of TR-IRAS spectra of NO on Pt(997) after an NO gas injection. (b) Change of the fractional coverage of each NO species as a function of the elapsed time. (Coverage: $0.02 \mathrm{ML}$, temperature: $76 \mathrm{~K}$.).

and the coverage of OT, HT, and BS species as a function of elapsed time. The coverage of each species is defined as the ratio of the number of NO molecules at each site to the number of $\mathrm{Pt}$ atoms. The amount of OT species decreases and the amount of HT and BS species increases. The coverage change is estimated from the change of the peak intensities. The details are as follows: since the extinction coefficient of IR absorption by $\mathrm{N}-\mathrm{O}$ stretching depends on the NO adsorption sites, the ratio of the extinction coefficient of OT species to that of HT and BS species is necessary to convert the peak intensity to the coverage. The coverage, whereby NO fully occupies the step site and the other sites are completely empty, is $0.056 \mathrm{ML}$. Note that the distance between neighboring NO molecules is about $5.5 \AA$, twice of the lattice constant of Pt substrate. Here, $1 \mathrm{ML}$ is defined as the ratio of the number of adsorbed $\mathrm{NO}$ molecules to the number of platinum atoms. We determined the coverage of BS species by comparing the peak intensity to that of 0.056 ML BS species (When all BS sites are occupied, the nearest-neighbor distance between NO molecules is about $5.5 \AA$. The dipole-dipole interaction should be considered in IRAS spectra and the peak could be slightly blue-shifted. However, we assume that the integrated intensity does not deviate from the simple proportional relation $I_{\mathrm{BS}}=$ $C_{\mathrm{BS}} N_{\mathrm{BS}}$ so much). In addition, the coverage of HT and OT species is also estimated by using the ratio of extinction coefficients. The total coverage of $\mathrm{NO}$ on $\mathrm{Pt}(997)$ is estimated by heating the substrate up to $150 \mathrm{~K}$. After heating, all NO molecules migrate to BS sites and the only peak of BS species remains in the IRAS spectrum. Note that neither desorption nor dissociation of NO molecules occurs at $150 \mathrm{~K}$ [11-13]. 


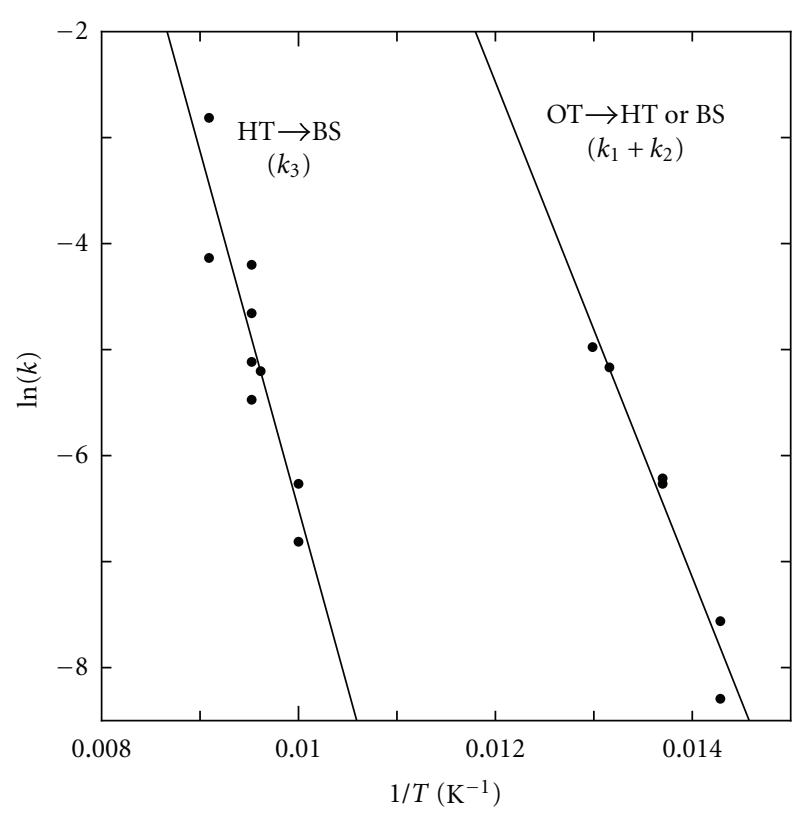

FIgURE 6: Arrhenius plots of the hopping processes of $k_{1}+k_{2}$ and $k_{3} . k_{1}+k_{2}$ and $k_{3}$ are the rate constants to escape from an OT and an HT site, respectively.

Rate constants of the site-to-site hopping from the OT site to the HT and BS site are defined as $k_{1}$ and $k_{2}$. The coverage change is analyzed based on first-order reaction kinetics, and $k_{1}$ and $k_{2}$ can be estimated from the following equations:

$$
\begin{gathered}
\frac{d \theta_{\mathrm{OT}}}{d t}=-\left(k_{1}+k_{2}\right) \theta_{\mathrm{OT}}, \\
\frac{d \theta_{\mathrm{HT}}}{d t}=k_{1} \theta_{\mathrm{OT}}, \\
\frac{d \theta_{\mathrm{BS}}}{d t}=k_{2} \theta_{\mathrm{OT}} .
\end{gathered}
$$

$\theta_{i}(t)(i=\mathrm{OT}, \mathrm{HT}$ and BS) is the coverage of OT, HT, and BS species, respectively. Similar experiments were carried out in the temperature range from $70 \mathrm{~K}$ to $77 \mathrm{~K}$, and the activation energies and preexponential factors of $k_{1}$ and $k_{2}$ are estimated. An Arrhenius plot for $k_{1}+k_{2}$, which is the rate constant to escape from the OT site, is shown in Figure 6. The activation barrier and the preexponential factor are estimated to be about $200 \mathrm{meV}$ and $2.0 \times 10^{-11} \mathrm{~s}^{-1}$, respectively. Since adsorbed NO molecules still remain in the terrace region in this temperature range, $k_{1}$ and $k_{2}$ are the rate constants of site-to-site hopping from the OT site to the neighboring HT or BS site, and long-range surface diffusion does not occur. Note that the $\theta_{\mathrm{HT}}(\infty) / \theta_{\mathrm{BS}}(\infty)$ is about $8 \sim 9$. We think that it depends on the terrace width of $\operatorname{Pt}(997)$, whose terrace is constructed by 9 atomic rows. This means that OT species only migrate to the nearest neighbor site (HT or BS), and the destinations depend on the initial position. OT species next to the step can only reach the step site. If a stepped $\operatorname{Pt}(111)$ surface, which has a longer terrace width than $\operatorname{Pt}(997)$, is adopted, larger $\theta_{\mathrm{HT}}(\infty) / \theta_{\mathrm{BS}}(\infty)$ value than $8 \sim 9$ would be expected.

Figure 7 shows a series of TR-IRAS spectra of NO on $\mathrm{Pt}(997)$ at $105 \mathrm{~K}$, and the coverage change of HT and BS species as a function of elapsed time. As the elapsed time increases, the peak intensity of HT species decreases and that of BS species increases. Long-range surface diffusion occurs at $105 \mathrm{~K}$ and all NO molecules migrate to the step edge. KMC simulation, which is described in Section 2, is adopted in the analysis. The parameters are the initial coverage $\left(\theta_{\text {total }}(0)\right)$, the rate constant to escape from the HT site $\left(k_{3}\right)$, and the interaction energy between two NO molecules in the $p(2 \times 2)$ superstructure. From the analysis, we found that the model with no ES barrier shown in Figure 2(b) is much better than that with the infinite ES barrier model shown in Figure 2(c). The solid line in Figure 7(b) shows the result of KMC simulation based on the first model. The second model cannot fit the experimental data with any parameters; thus, fitting by the second model is not shown here. Despite the fact that hopping from the HT site on the lower terrace to the BS site involves ascending the monoatomic step, the first model, which has no ES barrier, is better as a model of NO diffusion on $\mathrm{Pt}(997)$. This is because of the stable adsorption site at the HS site. The details are discussed later. Similar TR-IRAS measurements are conducted in the range from $100 \mathrm{~K}$ to $110 \mathrm{~K}$, and an Arrhenius plot for $k_{3}$ is shown in Figure 6. The activation barrier and the preexponential factor are estimated to be about $290 \mathrm{meV}$ and $6.5 \times 10^{11} \mathrm{~s}^{-1}$, respectively.

Figure 8 shows IRAS spectra of $\mathrm{NO}$ on $\operatorname{Pt}(997)$ as a function of heating temperature. After heating the substrate to $45 \mathrm{~K}$, the peak of the HS species diminishes, and only that of the BS species increases, and the others do not change. This indicates that all NO molecules at HS sites migrate to BS sites by ascending the monoatomic step, which supports the fact that no ES barrier is discussed above. We roughly estimated the barrier height as follows: since the HS species is observable at $40 \mathrm{~K}$, the accommodation time of the HS site at $40 \mathrm{~K}$ is similar or longer than the measurement time at least. On the other hand, this diminishes immediately at $45 \mathrm{~K}$; thus, the accommodation time of the HS site at $45 \mathrm{~K}$ is smaller than the measurement time. Therefore, assuming that the preexponential factor is $10^{11} \mathrm{~s}^{-1}$, the activation barrier to hopping from the HS site to the BS site is in the range from $110 \mathrm{meV}$ to $120 \mathrm{meV}$.

3.3. Transient Diffusion Process of NO on Pt(997). Thermal diffusion of NO on $\mathrm{Pt}(997)$ does not occur at $11 \mathrm{~K}$ (Since the shape of the IRAS spectrum at $11 \mathrm{~K}$ did not change at all with increasing the elapsed time, we concluded that the site-to-site hopping of adsorbed NO molecules did not occur at $11 \mathrm{~K}$.); the distribution of the adsorbed NO molecules on $\mathrm{Pt}(997)$ at $11 \mathrm{~K}$ is determined by the first collision point from the gas phase and the distance of transient diffusion from this collision point. We can estimate the distance of the transient diffusion of $\mathrm{NO}$ on $\mathrm{Pt}(997)$ from a series of IRAS spectra shown in Figure 3 [14]. We define the extinction coefficient of an adsorbed NO molecule as $C_{i}(i=\mathrm{HS}, \mathrm{HT}, \mathrm{BS}, \mathrm{OT})$. In 


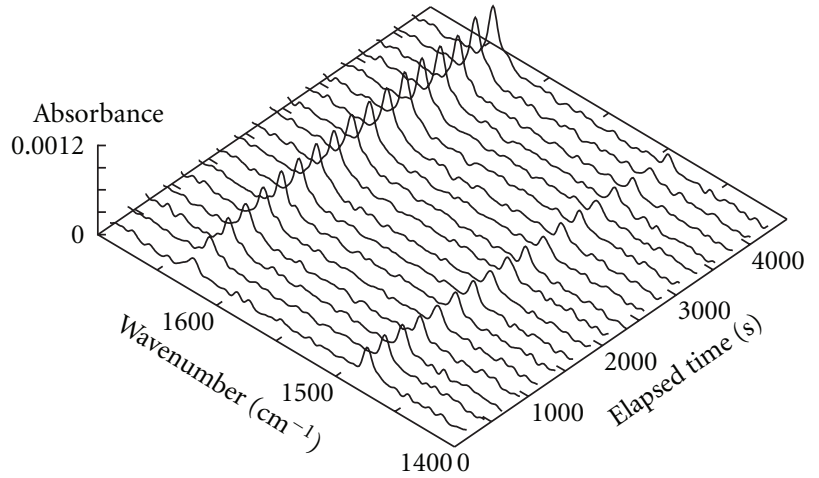

(a)

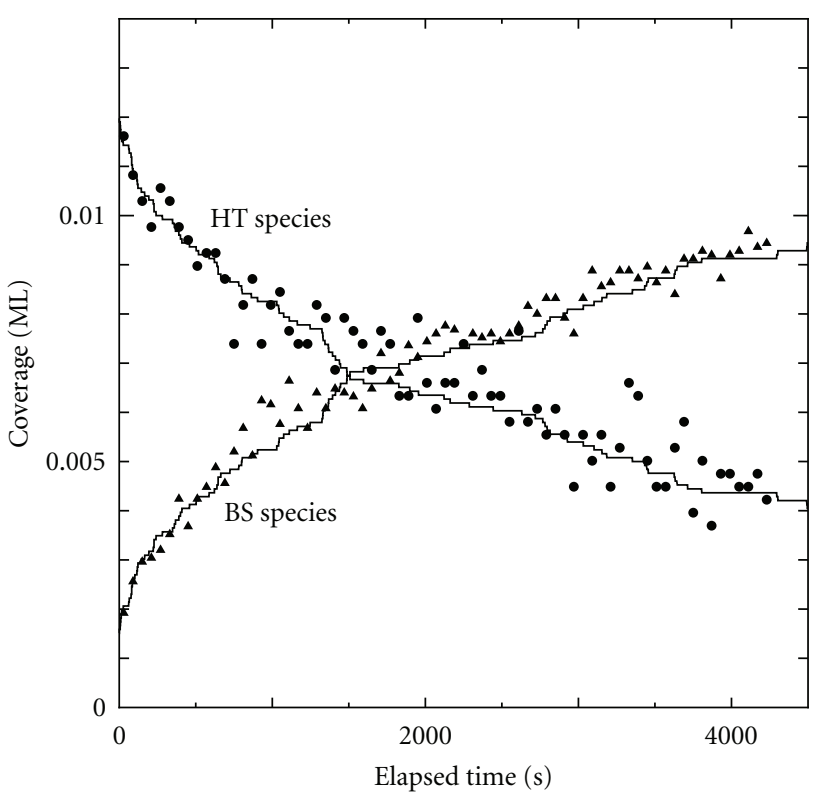

(b)

Figure 7: (a) A series of TR-IRAS spectra of NO on Pt(997) after an NO gas injection. (b) Change of the fractional coverage of each NO species as a function of the elapsed time. (Coverage: $0.017 \mathrm{ML}$, temperature: $105 \mathrm{~K}$.).

all of the spectra of Figure 3, the total coverage of adsorbed $\mathrm{NO}$ is the same

$$
\begin{array}{r}
\frac{I_{\mathrm{HS}}(11)}{C_{\mathrm{HS}}}+\frac{I_{\mathrm{HT}}(11)}{C_{\mathrm{HT}}}+\frac{I_{\mathrm{BS}}(11)}{C_{\mathrm{BS}}}+\frac{I_{\mathrm{OT}}(11)}{C_{\mathrm{OT}}} \\
=\frac{I_{\mathrm{HT}}(70)}{C_{\mathrm{HT}}}+\frac{I_{\mathrm{BS}}(70)}{C_{\mathrm{BS}}}+\frac{I_{\mathrm{OT}}(70)}{C_{\mathrm{OT}}} \\
=\frac{I_{\mathrm{HT}}(90)}{C_{\mathrm{HT}}}+\frac{I_{\mathrm{BS}}(90)}{C_{\mathrm{BS}}}=\frac{I_{\mathrm{BS}}(150)}{C_{\mathrm{BS}}},
\end{array}
$$

where $I_{i}(T)=C_{i} N_{i}(T)$ is the peak intensity of adsorbed NO at site $i$, and $N_{i}(T)$ is the number of adsorbed NO molecules at site $i$. From these equations and the spectra of Figure 1, the ratio of extinction coefficients is estimated to be $C_{\mathrm{BS}} / C_{\mathrm{HT}}=$ 3.3 and $C_{\mathrm{BS}} / C_{\mathrm{OT}}=2.2$, respectively. We could not estimate $C_{\mathrm{BS}} / C_{\mathrm{HS}}$ from the spectrum of Figure 3(a). (Comparing the spectrum (a) with (c) in Figure 7, the peak position of BS species is slightly different. It seems that this is caused by the change of adsorption state of BS species, and the $C_{\mathrm{BS}}$ is also different between $11 \mathrm{~K}$ and $40 \mathrm{~K}$.) However, the ratio of the number of NO in the step region (HS and BS) and that of $\mathrm{NO}$ in the terrace region (HT and OT) at $11 \mathrm{~K}$ is same as at $45 \mathrm{~K}$, at which HS species diminishes. From the ratio of peak intensities at $45 \mathrm{~K}$, the ratio of $\mathrm{NO}$ on the step to $\mathrm{NO}$ on the terrace is about $1: 6.1$. Based on a previous study of $\mathrm{CO}$ on $\mathrm{Pt}(997)$ [14], the simple model shown in Figure 9 is used to estimate the mean distance of transient diffusion. Therefore, the mean distance of transient diffusion is about $4.1 \AA$.

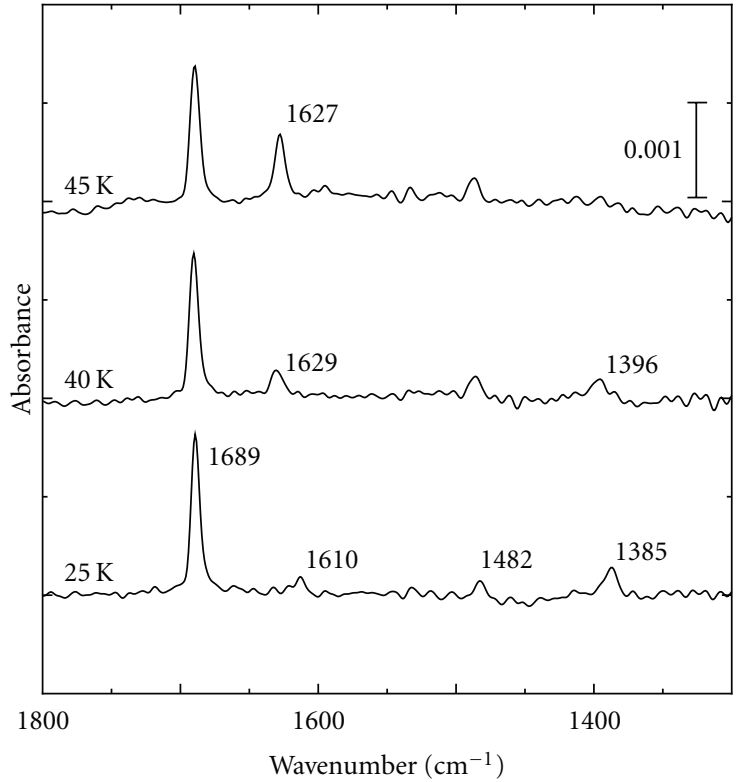

FIGURE 8: IRAS spectra of 0.027 ML NO on Pt(997) as a function of the heating temperature. NO molecules were adsorbed at $11 \mathrm{~K}$.

\section{Comparison with Other Studies}

In this section, we discuss the above experimental results by comparison with the previous studies. In the He Atom Scattering (HAS) study of $\mathrm{NO}$ on $\mathrm{Pt}(111)$ [30], the activation barrier was estimated to be $510 \mathrm{meV}$, which is larger than our result $(290 \mathrm{meV})$. However, their measurement was carried out on the macroscopic region and at higher temperatures 


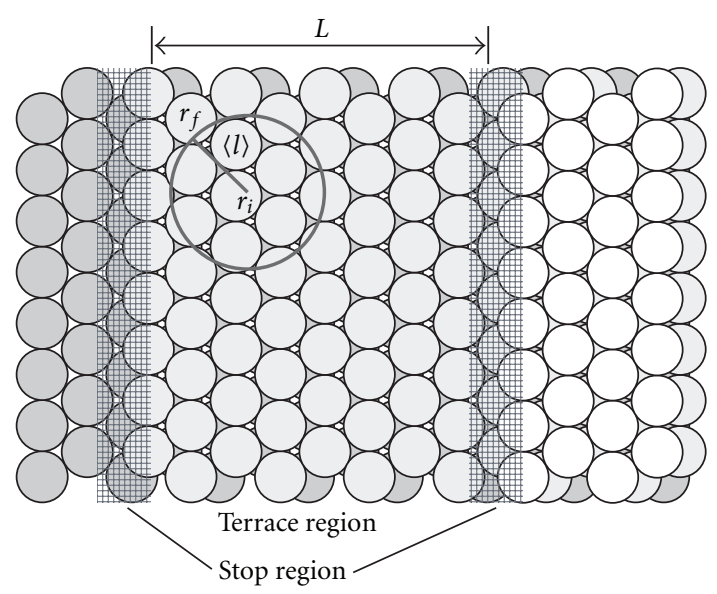

Figure 9: A schematic drawing for the estimation of transient diffusion length of $\mathrm{NO}$ on $\mathrm{Pt}(997)$. The shadowed regions and the other regions are regarded as the step region and the terrace region, respectively. $r_{i}$ and $r_{f}$ are an initial collision site and a final adsorption site, respectively. By considering the ratio of NO on the step to that on the terrace, the transient diffusion length $\langle l\rangle$ can be estimated based on Buffon's needle problem [2, 14].

$(310 \sim 360 \mathrm{~K})$. Therefore, hopping across the step may be included and it could be the rate-limiting process. In addition, the dissociation of $\mathrm{NO}$ at the step site should occur above $200 \mathrm{~K}$ [11-13]. It is reasonable that the barrier height, $290 \mathrm{meV}$, is smaller than the HAS result.

In a previous IRAS and DFT study of NO on Pt(110) [25], the authors estimated the activation barrier to NO hopping. Hopping from the on-top site to the bridge site on the Pt atomic row is about $110 \mathrm{meV}$, and that from the hollow site, which is next to the bridge site on the atomic row to the bridge site on the atomic row, is about $230 \mathrm{meV}$. Their results are in agreement with our results.

The distance of transient diffusion of $\mathrm{NO}$ on $\mathrm{Pt}(997)$ is about $4.1 \AA$. On the other hand, that of $\mathrm{CO}$ on $\mathrm{Pt}(997)$ is about $6.8 \AA$ [14], which is longer than that of NO. Physisorbed species, such as noble gas, transiently migrates in the order of hundreds of $\AA$. CO and NO molecules chemisorb on the Pt surface, and their bonding is stronger than the physisorbed species. Chemisorbed $\mathrm{CO}$ and NO dissipate their adsorption energy to the substrate via mainly substrate phonon and electron-hole pair excitations. This results in a shorter length of transient diffusion. Comparing $\mathrm{NO}$ with $\mathrm{CO}$, the transient diffusion length of $\mathrm{NO}$ is shorter than that of CO. This is caused by stronger coupling between $\mathrm{NO}$ and the Pt substrate. NO has an unpaired electron in the $2 \pi$ orbital, which is empty in the case of $\mathrm{CO}$. This unpaired electron makes the energy transfer from NO to the substrate efficient when NO molecules migrate on the surface. In addition, the shape of PES of NO is more complex than that of CO. The stable adsorption site of CO on $\mathrm{Pt}(997)$ is only an on-top site at low coverage, and the activation barrier to $\mathrm{CO}$ hopping is about $190 \mathrm{meV}$ [20-22]. On the other hand, the number of stable adsorption sites for NO is larger than for $\mathrm{CO}$, and the activation barrier to NO hopping is higher than

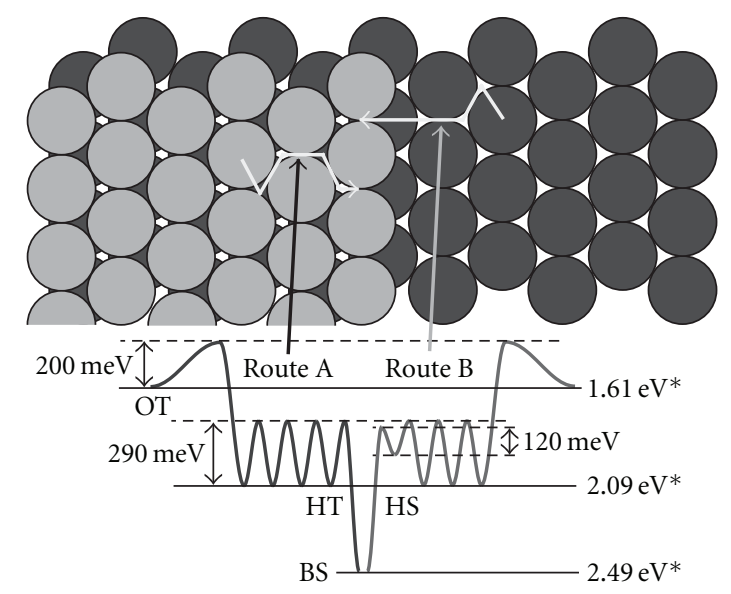

Figure 10: Potential energy surface of NO on $\mathrm{Pt}(997)$. ${ }^{*}$ The adsorption energy is the result from the previous DFT studies $[10,16]$.

that of CO hopping. Therefore, this complicated PES shape results in the short length of NO transient diffusion.

Based on the IRAS experiments and the KMC simulation, PES of NO on Pt(997) is clarified. We conclude that the HT species can approach the step from both the upper and lower terraces. Although we cannot rule out the asymmetry of PES at the step, the NO molecules can easily migrate from a HS site to a BS site. This behavior is different from $\mathrm{CO}$ on a stepped Pt (111) surface, which has a large ES barrier [20$22]$. NO molecules migrate to the upper step by climbing the activation barrier of about $120 \mathrm{meV}$. Figure 10 shows a schematic drawing of the routes for NO molecules from the terrace to the step and the shape of potential energy.

\section{Summary}

We have presented the PES of NO on $\mathrm{Pt}(997)$; the microscopic diffusion process of $\mathrm{NO}$ molecules on $\mathrm{Pt}(997)$ and the features of PES of NO, which are related to surface diffusion, are discussed. From the gas phase, NO collides with the Pt surface and transiently diffuses and reaches a stable adsorption site. The mean length of transient diffusion is about $4.1 \AA$. After the transient diffusion, thermal diffusion occurs at higher temperatures. First, NO molecules at HS sites thermally migrate up to the BS site. The activation barrier is about $120 \mathrm{meV}$. Secondly, OT species migrate to the neighboring HT or BS site. The activation barrier and the preexponential factor are about $200 \mathrm{meV}$ and $2.0 \times 10^{11} \mathrm{~s}^{-1}$, respectively. Thirdly, the HT species start to diffuse on the terrace to the BS site, which is the most stable for NO. The activation barrier and the preexponential factor are about $290 \mathrm{meV}$ and $6.5 \times 10^{11} \mathrm{~s}^{-1}$, respectively. In addition, there is little ES effect on $\mathrm{NO}$ on $\mathrm{Pt}(997)$.

\section{References}

[1] J. V. Barth, "Transport of adsorbates at metal surfaces: from thermal migration to hot precursors," Surface Science Reports, vol. 40, no. 3, pp. 75-149, 2000. 
[2] W. A. Brown and D. A. King, "NO chemisorption and reactions on metal surfaces: a new perspective," Journal of Physical Chemistry B, vol. 104, no. 12, pp. 2578-2595, 2000.

[3] H. Ibach and S. Lehwald, "Analysis of adsorption processes and surface reactions by vibration spectroscopy: adsorption of no on Pt(111)," Surface Science, vol. 76, no. 1, pp. 1-12, 1978.

[4] J. L. Gland and B. A. Sexton, "Nitric oxide adsorption on the Pt(111) surface," Surface Science, vol. 94, no. 2-3, pp. 355-368, 1980.

[5] B. E. Hayden, "An infra-red reflection absorption study of the adsorption of NO on Pt(111)," Surface Science, vol. 131, no. 2-3, pp. 419-432, 1983.

[6] J. Yoshinobu and M. Kawai, "Adsorption and dimer formation of nitrogen monoxide on $\mathrm{Pt}(111)$ at low temperatur," Chemistry Letters, vol. 24, no. 7, p. 605, 1995.

[7] I. Villegas, R. Gomez, and M. J. Weaver, "Nitric oxide as a probe adsorbate for linking $\mathrm{Pt}(111)$ electrochemical and model ultrahigh-vacuum interfaces using infrared spectroscopy," Journal of Physical Chemistry, vol. 99, no. 40, pp. 14832-14839, 1995.

[8] M. Matsumoto, K. Fukutani, T. Okano et al., "Study of the adsorption structure of $\mathrm{NO}$ on $\mathrm{Pt}(111)$ by scanning tunneling microscopy and high-resolution electron energy-loss spectroscopy," Surface Science, vol. 454, no. 1, pp. 101-105, 2000.

[9] M. Matsumoto, N. Tatsumi, K. Fukutani, and T. Okano, "Dynamical low-energy electron diffraction analysis of the structure of nitric oxide on $\operatorname{Pt}\left(\begin{array}{lll}1 & 1 & 1\end{array}\right)$," Surface Science, vol. 513, no. 3, pp. 485-500, 2002.

[10] H. Aizawa, Y. Morikawa, S. Tsuneyuki, K. Fukutani, and T. Ohno, "A density-functional study of the atomic structures and vibrational spectra of NO/Pt(l 111$)$," Surface Science, vol. 514, no. 1-3, pp. 394-403, 2002.

[11] V. K. Agrawal and M. Trenary, "An infrared study of NO adsorption at defect sites on Pt(111)," Surface Science, vol. 259, no. 1-2, pp. 116-128, 1991.

[12] R. J. Mukerji, A. S. Bolina, W. A. Brown, Z. P. Liu, and P. Hu, "The temperature dependence of the adsorption of NO on Pt(211): a RAIRS and DFT investigation," Journal of Physical Chemistry B, vol. 108, no. 1, pp. 289-296, 2004.

[13] E. H. G. Backus, A. Eichler, M. L. Grecea, A. W. Kleyn, and M. Bonn, "Adsorption and dissociation of NO on stepped Pt(533)," Journal of Chemical Physics, vol. 121, no. 16, pp. 7946-7954, 2004.

[14] J. Yoshinobu, N. Tsukahara, F. Yasui, K. Mukai, and Y. Yamashita, "Lateral displacement by transient mobility in chemisorption of CO on Pt(1997)," Physical Review Letters, vol. 90, no. 24, pp. 248301-248301, 2003.

[15] N. Tsukahara, K. Mukai, Y. Yamashita, and J. Yoshinobu, "Microscopic diffusion processes of NO on the Pt(997) surface," Journal of Chemical Physics, vol. 128, no. 5, Article ID 054701, 2008.

[16] N. Tsukahara, K. Mukai, Y. Yamashita, J. Yoshinobu, and H. Aizawa, "Adsorption states of NO on the $\operatorname{Pt}\left(\begin{array}{lll}1 & 1 & 1\end{array}\right)$ step surface," Surface Science, vol. 600, no. 17, pp. 3477-3483, 2006.

[17] N. Tsukahara, K. Mukai, Y. Yamashita, and J. Yoshinobu, "Search for adsorption potential energy minima of NO on Pt(9 9 7) at 11 K," Surface Science, vol. 600, no. 18, pp. 3560 $3563,2006$.

[18] K. Binder and D. P. Landau, "Phase diagrams and critical behavior in Ising square lattices with nearest- and next-nearestneighbor interactions," Physical Review B, vol. 21, no. 5, pp. 1941-1962, 1980.
[19] M. Tringides and R. Gomer, "A Monte Carlo study of oxygen diffusion on the (110) plane of tungsten," Surface Science, vol. 145, no. 1, pp. 121-144, 1984.

[20] J. E. Reutt-Robey, D. J. Doren, Y. J. Chabal, and S. B. Christman, "Microscopic co diffusion on a PT(111) surface by time-resolved infrared spectroscopy," Physical Review Letters, vol. 61, no. 24, pp. 2778-2781, 1988.

[21] J. E. Reutt-Robey, Y. J. Chabal, D. J. Doren, and S. B. Christman, "CO diffusion on $\mathrm{Pt}(111)$ by time-resolved surface infrared spectroscopy," Journal of Vacuum Science \& Technology A, vol. 7, no. 3, pp. 2227-2234, 1989.

[22] J. E. Reutt-Robey, D. J. Doren, Y. J. Chabal, and S. B. Christman, "CO diffusion on $\mathrm{Pt}(111)$ with time-resolved Infraredpulsed molecular beam methods: critical tests and analysis," The Journal of Chemical Physics, vol. 93, no. 12, pp. 9113-9129, 1990.

[23] G. Ehrlich and F. G. Hudda, "Atomic view of surface selfdiffusion: tungsten on tungsten," The Journal of Chemical Physics, vol. 44, no. 3, pp. 1039-1049, 1966.

[24] R. L. Schwöebel and E. J. Shipsey, "Step motion on crystal surfaces," Journal of Applied Physics, vol. 37, no. 10, pp. 36823686, 1966.

[25] W. A. Brown, R. K. Sharma, Q. Ge, and D. A. King, "Lateral potential energy surfaces for molecular chemisorption on metals from experiment and theory: NO on Pt $\{110\}-(1 \times 2)$," Chemical Physics Letters, vol. 299, no. 3-4, pp. 253-259, 1999.

[26] C. Uebing and R. Gomer, "A Monte Carlo study of surface diffusion coefficients in the presence of adsorbate-adsorbate interactions. I. Repulsive interactions," The Journal of Chemical Physics, vol. 95, no. 10, pp. 7626-7635, 1991.

[27] C. Uebing and R. Gomer, "A Monte Carlo study of surface diffusion coefficients in the presence of adsorbate-adsorbate interactions. II. Attractive interactions," The Journal of Chemical Physics, vol. 95, no. 10, pp. 7636-7640, 1991.

[28] C. Uebing and R. Gomer, "A Monte Carlo study of surface diffusion coefficients in the presence of adsorbate-adsorbate interactions. III. Repulsive nearest-neighbor and attractive next-nearest-neighbor interactions," The Journal of Chemical Physics, vol. 95, no. 10, pp. 7641-7647, 1991.

[29] C. Uebing and R. Gomer, "A Monte Carlo study of surface diffusion coefficients in the presence of adsorbate-adsorbate interactions. IV. Attractive nearest-neighbor and repulsive next-nearest-neighbor interactions," The Journal of Chemical Physics, vol. 95, no. 10, pp. 7648-7652, 1991.

[30] M. Croci, C. Félix, G. Vandoni, W. Harbich, and R. Monot, "Chemisorption and macroscopic diffusion of NO on Pt(111)," Surface Science, vol. 307-309, pp. 460-464, 1994. 


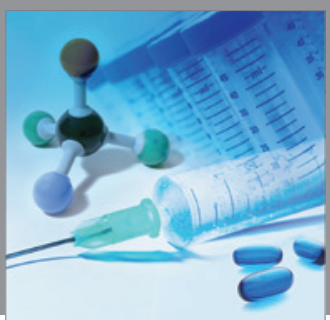

International Journal of

Medicinal Chemistry

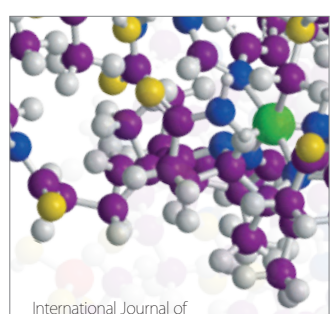

Carbohydrate Chemistry

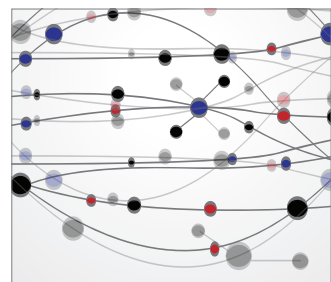

The Scientific World Journal
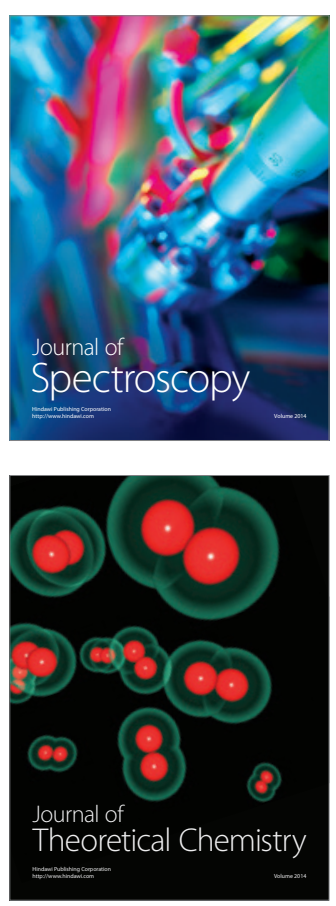
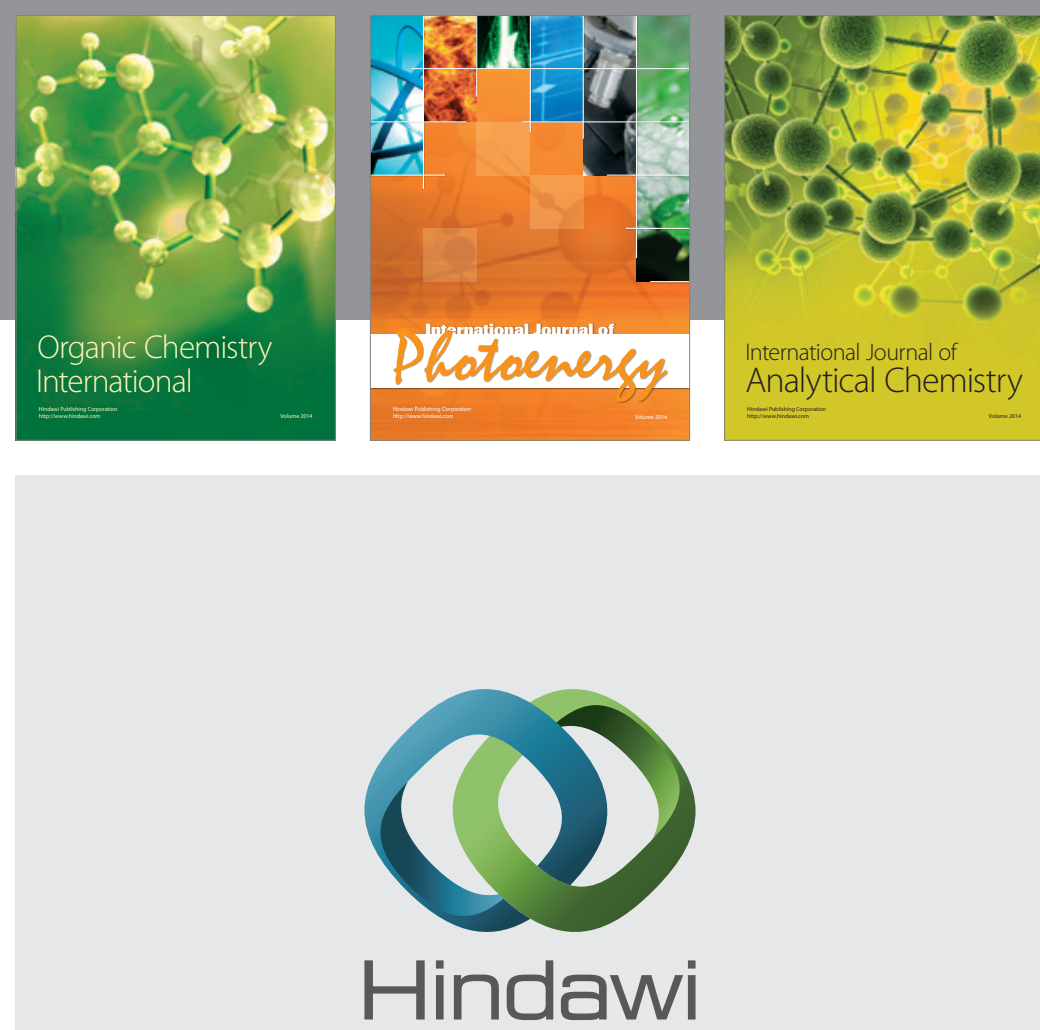

Submit your manuscripts at

http://www.hindawi.com
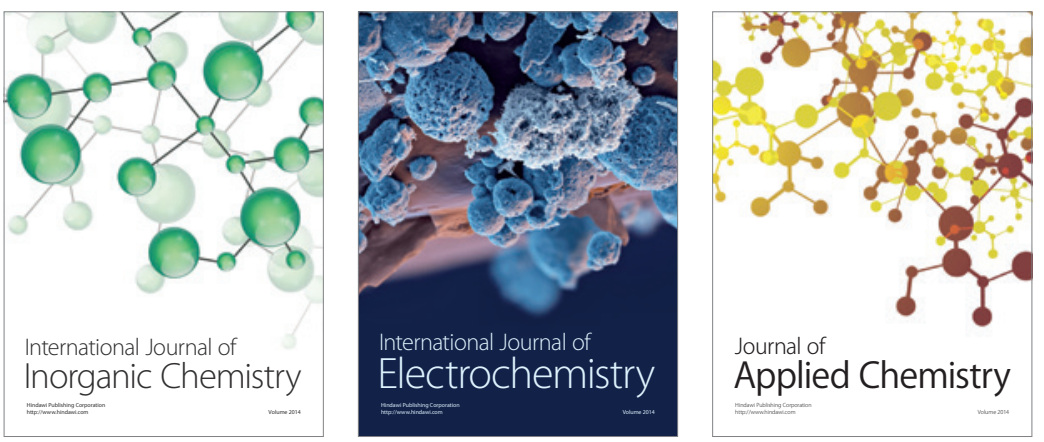

Journal of

Applied Chemistry
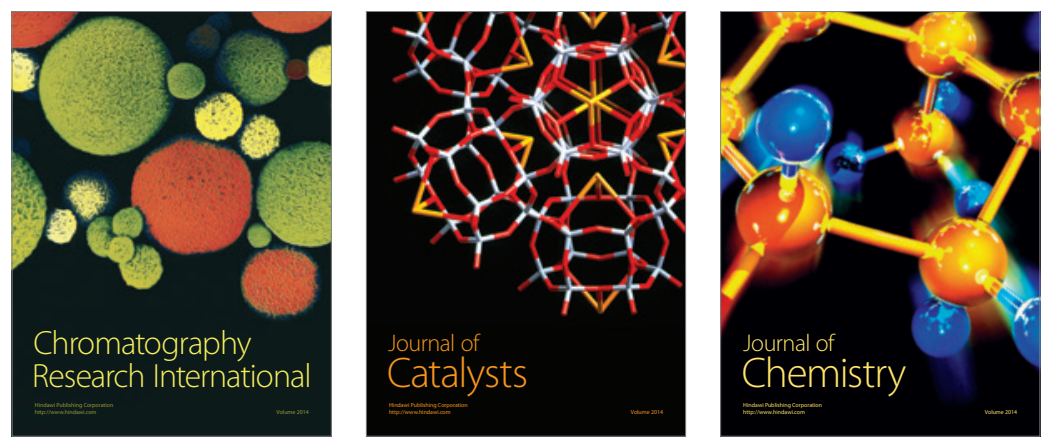
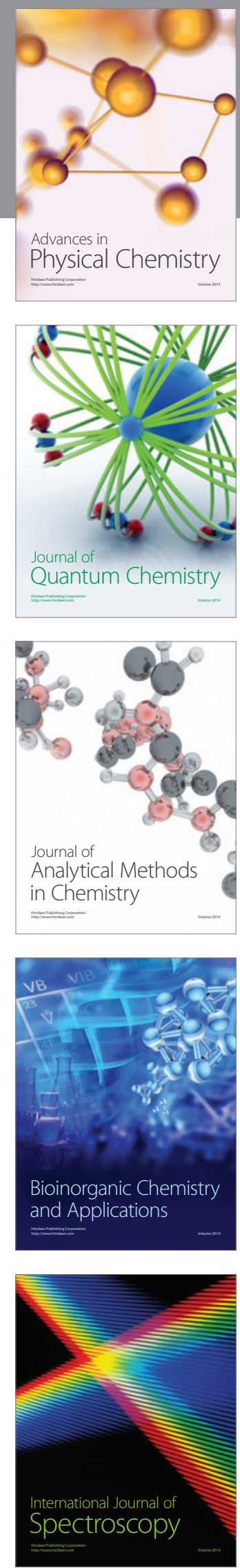